\title{
Francisco Suárez y las matemáticas. Notas sobre su carácter científico según las Disputaciones Metafísicas I
}

\author{
José María Felipe Mendoza \\ Universidad Nacional de Cuyo / CONICET \\ josefelipemendoza@hotmail.com \\ https://orcid.org/0000-0002-1881-0947
}

Resumen: El presente trabajo propone una introducción a la doctrina de la ciencia matemática de Francisco Suárez según sus Disputaciones Metafisicas I. El contexto de la investigación aborda la noción de objeto adecuado, una teoría de la abstracción y el orden de las ciencias especulativas en general. Por ello, para mejor comprender las ciencias matemáticas, es necesario observar su relación con la metafísica, delimitando no solo su fundamento, sino también su horizonte especulativo.

Palabras clave: Francisco Suárez; metafísica; matemática; objeto adecuado; abstracción

Abstract: "Francisco Suarez and Mathematics. Remarks on its Scientific Character
according to Disputationes Metaphysicae I". The present work proposes a brief
introduction to the mathematical doctrine of Francisco Suarez according to his
Disputationes Metaphysicae I. The context of his researching deals with the notion
of obiectum aedecuatum, a theory of abstraction and the order of sciences in general
terms. Therefore, in order to better understanding the mathematical sciences, it's
necessary to observe its relationship with metaphysics and define the ground and
the limits of mathematical philosophy.

Keywords: Francisco Suárez; metaphysics; mathematics; obiectum aedecuatum; abstraction 


\section{Preliminares}

En el año de 1597 se publicaron las Disputationes Metaphysicae de Francisco Suárez [S.J.] en la Universidad de Salamanca ${ }^{2}$. Una obra de erudición asombrosa que ponía de manifiesto un aristotelismo renacentista claramente distante de aquellos propios de la Edad Media, sin que por ello se dejase de asumir todo cuanto parecía haberse dicho ${ }^{3}$. Suárez fue un filósofo genuinamente moderno que ha pensado textos antiguos, medievales y renacentistas. Su escolástica es barroca ${ }^{4}$ y su conocimiento de la filosofia emana de las variantes aristotélicas.

La D.M. I -texto al que nos abocaremos- tiene por objetivo fijar la razón de las ciencias especulativas, fundamentar qué es la ciencia metafisica y aclarar a qué ciencias se extiende legítimamente. Sus páginas muestran un movimiento especulativo de continuidad y ruptura con la tradición. No constituyen una glosa al tratado aristotélico de la Metafísica al modo que era frecuente en la Edad Media escolástica, y si lo reorganizan en tópicos fundamentales. En dicha

\footnotetext{
1 Se sigue aquí la edición de Suarez, F., Disputaciones Metafisicas, Madrid: Gredos, 1960-1966, y cuyo modo de citar es D.M., a lo que le sigue la numeración de la disputación, la sección y el pasaje. A ello conviene añadir que este estudio presenta la totalidad de casos en que la noción de matemática es mencionada por el Granadino y la ausencia en la bibliografia secundaria de estudios específicos sobre la mentada ciencia.

2 Pueden consultarse para el contexto histórico de las universidades y el saber en general los siguientes textos: Hale, J.R., La Europa del Renacimiento 1480-1520, Madrid: Siglo XXI, 2016, pp. 293-336; Parker, G., Europa en crisis 1598-1648, Madrid: Siglo XXI, 2017, pp. 354-373; Belda Plans, J., La escuela de Salamanca y la renovación de la teología en el siglo XVI, Madrid: BAC, 2000; Poncela González, Á., "Aristóteles y los jesuitas. La génesis corporativa de los cursus philosphicus”, en: Cauriensia, v. VI (2011), pp. 65-101. Para la vida y la obra de Suárez, donde se destacan tanto la extraordinaria erudición y prolijidad de sus escritos en teología, filosofia y derecho como así también sus cátedras en las universidades prestigiosas del momento: Valla-

294 dolid, Segovia, Ávila, Roma, Alcalá, Salamanca, Coímbra y Lisboa, pueden consultarse Prieto López, L. J., Suárez y el destino de la metafisica, Madrid: BAC, 2013, pp. 17-100; Salas V. y R. Fastiggi, "Francisco Suárez, the Man and his Work", en: Salas V. y R. Fastiggi (eds.), A Companion to Francisco Suárez, Lieden-Boston: Brill, 2014, pp. 1-28.

3 Gilson, E., El ser y los filósofos, Pamplona: Eunsa, 1979, p. 156: "Suárez goza de un conocimiento tal de la filosofia medieval como para avergonzar a cualquier historiador moderno del pensamiento medieval. En todas y cada una de las cuestiones parece conocerlo todo y a todo el mundo".

4 Esposito, C., "Suárez, filósofo barroco", en: Cauriensia, XII (2017), p. 29: “debemos llegar a decir que es la experiencia de los jesuitas la que ilumina las raíces del barroco”.
} 
obra se observa un esfuerzo un tanto ecléctico ${ }^{5}$ de fijar la razón de la metafísica. Y de alli la erudición del filósofo Granadino. En las D. M. I aparecen Alejandro de Afrodisia, Temistio, Simplicio, Proclo, Averroes, Alejandro de Hales, Alberto Magno, Tomás de Aquino, Egidio Romano, Duns Scoto y Buridano, entre otros ${ }^{6}$. Sin embargo, la erudición de Suárez no parece tener en particular consideración otros desarrollos especulativos distantes del aristotelismo. Platón es citado en contadas ocasiones en orden a su conversión con Aristóteles ${ }^{7}$, mientras que la tradición platónica -donde la matemática encontró un desarrollo más atento- habria corrido la misma fortuna. El mundo de las ideas, la matemática y los experimentos no halló cauce en el Dr. Eximio ${ }^{8}$. Pero no por ello puede avalarse una posición que señale al Granadino como un autor de mentalidad netamente medieval. La segunda escolástica es fruto de la escolástica crítica, y el ingenio de Suárez emerge de su revitalización. Así, por ejemplo, se vale del humanista Juan Argirópulo ${ }^{9}$ para las traducciones de los textos griegos, y especialmente de Aristóteles, antes que aquellas medievales de Boecio y Moerbeke.

5 Rábade Romeo, S., "La metafisica de Suárez: subjetivización y dinamismo", en: Cuadernos salmantinos de filosofia, 30 (2003), p. 145.

${ }_{6}$ Después de la condena parisina del año de 1277 por el obispo Esteban Tempier, el aristotelismo en parte se mantiene, y en parte se supera mediante discusión de sus tesis físicas, lógicas y metafisicas. El s. XIV produce un cambio de enfoque en la realidad, cuya manifestación más evidente aparece en los modos de comprensión de las ciencias especulativas. Según este viraje, la revolución científica en tales ámbitos pone de manifiesto el enfrentamiento entre los antiqui y los moderni. Tomás de Aquino quedó entre los antiqui y Duns Scoto entre los moderni, quien, junto con Ockham y Buridan, fueron gestando un nueva intelección de lo real cuyas consecuencias en teología y filosofia alcanzaron tanto a Cayetano como a Suárez. Cf. Gilson, E. y Th. Langan, Filosofia moderna, Barcelona: Emecé, 1963, pp. 3-7; Fabro, C., Historia de la filosofia I, Madrid: Rialp, 1965, pp. 557-574; Verdú Berganza, I., "Aspectos generales del pensamiento en el siglo XIV", en: Anales del seminario de historia de la filosofia, 10 (1993), pp. 195-208; Florido, F.L., Las filosofias en la Edad Media. Crisis, controversias y condenas, Madrid: Biblioteca Nueva, 2010, pp. 233-289; Florido, F. L., "Una estructura filosófica en la historia de la filosofia", en: Anales del seminario de historia de la filosofia, 17 (2000), pp. 195-216; y Lohr, Ch.H., "Del aristotelismo medieval al aristotelismo renacentista", en: Patristica et Mediaevalia, v. XVII (1996), p. 13, donde se dice: "la base fundamental de la reinterpretación cristiana que llevó a cabo Suárez del pensamiento de Aristóteles reside en la relación entre la realidad finita y el infinito poder creador de Dios".

7 Suárez, F., D.M. I, V, 37: "quia ergo hoc est potissimum et difficillimum in scientia, et quia ipsamet dialéctica... nec aliud sensit plato, qui dialecticae nomine metaphysicam saepe intelligit". 8 El mundo físico halló un desarrollo científico en el lenguaje de la matemática aplicada, y no tanto una explicación metafísica del mismo. Cf. Crombie, A.C., Historia de la ciencia: de san Agustin a Galileo/2: siglos XIII-XVII, Madrid: Alianza Editorial, 1987, p. 113; y Koyré, A., Estudios galileanos, España: siglo XXI, 2009, p. 3.

Suárez, F., D.M. I, II, 23: "sed melius D. Thomas, et alii antiqui, quos Fonseca sequitur, ad hoc respondet, aliter construendo litteram Aristotelis, quae sic habet secundum versionem antiquam: entis in quantum ens quascumque species speculari unius est scientiae genere, et species specierum. in quibus verbis illa duo, et species specierum, aequivoca sunt; possunt enim coniungi, ita ut unum determinet aliud, et referantur ad ens, et ad species specierum entis, et hunc sensum significat clare versio argyropoli, et in eo procedit expositio data". Según el ejemplo seña- 
El Renacimiento de la cultura clásica y el Aristóteles latino y eclesiástico constituyen un aspecto importante del devenir de las ciencias metafísica, física y matemática ${ }^{10}$. Se debatía sobre todas ellas para lograr una inteligibilidad diferente ${ }^{11}$. Mismas ciencias, distinto fundamento y horizontes completamente diversos $^{12}$. Y aquí pensadores como Nicolás de Cusa y Copérnico, y el surgimiento de una nueva tradición astronómica que logró superar, sobre la base de Platón y Arquímides, entre muchos otros, las diversas interpretaciones aristotélicas que fueron naciendo con el transcurrir de los siglos XV y XVI ${ }^{13}$.

A su vez, los desarrollos especulativos en el ámbito de las matemáticas no parecen haber sido desconocidos por Suárez en su totalidad. Antes bien, el Dr. Eximio menciona cierta apertura a aspectos científicos más próximos a los desarrollos matemáticos de la época, como el texto de Proclo In primum Euclidis elementorum librum commentarii ${ }^{14}$. Pero todo ello es insuficiente en sí

lado, Suárez prefiere (i) la traducción de Argirópulo de la Metafisica de Aristóteles; (ii) observa la continuidad de interpretación entre Tomás de Aquino y la suya a través de una traducción no usada por Tomás; (iii) logra la convergencia entre las interpretaciones textuales -la suya, aquella de Tomás de Aquino y de Fonseca- por sobre las traducciones a la Metafisica de los antiguos (referencia a los teólogos previos a la condena de 1277); (iv) queda asentada la probabilidad de que Suárez se haya valido también de otras traducciones de Argirópulo en lo tocante a los textos del Estagirita.

${ }^{10}$ Crombie, A.C., Historia de la ciencia, pp. 99-100: "este movimiento literario realizó algunos servicios importantes a la ciencia... el servicio más inmediato fue el de proporcionar los medios de desarrollo de la ciencia matemática, y las traducciones y ediciones de textos... con mucho, las ediciones más numerosas de un autor fueron las de las obras de Aristóteles, acompañadas frecuentemente con las glosas de Averroes y de otros comentaristas".

${ }^{11}$ Koyré, A., Estudios galileanos, p. 5.

12 Ibid., p. 6: "la historia del pensamiento científico (fisico) de la Edad Media al Renacimiento... presenta tres etapas. Primero, física aristotélica. A continuación, física del ímpetus, inaugurada, como todo, por los griegos, pero elaborada fundamentalmente en el siglo XIV por la escuela parisiense de Buridan y Nicolás de Oresme; finalmente, fisica matemática, experimental, arquimediana o galileana".

${ }^{13}$ Crombie, A. C., Historia de la ciencia, p. 123: "los físicos del siglo XVI cambiaron progresivamente de las explicaciones físicas cualitativas de Aristóteles a las formulaciones matemáticas de Arquímides y al método experimental".

${ }^{14}$ Suarez, F., D. M. I, IV, 15: "et Proclus, lib. I Comment. in Euclid., c. 4, ubi etiam mathematicis scientiis ait metaphysicam suppeditare principia". En este sentido el platonismo de Proclo es interpretado en dirección al aristotelismo al quedar las matemáticas sujetas a la metafísica.

296 Conviene asimismo destacar que el texto de Euclides ya es citado v.g. por Tomás de Aquino (v.g. Metaphysica, lib. 3 1. 4 n. 12; Analitica Posteriora, lib. 1 1. 4 n. 13; De caelo, lib. 21.6 n. 7) en la misma dirección aristotelizante, mientras que pareciera desconocer el comentario de Proclo. De allí que el texto de Euclides y el comentario de Proclo signifiquen, en conjunto, un avance en el conocimiento de los textos científicos sobre matemática, aunque todo ello sea interpretado en el marco general del aristotelismo renacentista. Crombie, A.C., Historia de la ciencia, p. 99: "era inhabitual para los estudiantes de la universidad medieval ir más allá del libro primero de Euclides... algunos de estos autores griegos, como Euclides y Ptolomeo, habian sido estudiados en los siglos anteriores; otros, como Arquímides, Apolonio y Diofanto, estaban disponibles en traducciones antiguas, pero generalmente no estudiados". 
mismo y en lo que respecta a un diálogo fructifero, pues no hallamos acopio de los movimientos académico-filosóficos y humanistas que propiciaban una física, una matemática y una astronomía nuevas ${ }^{15}$.

El conocimiento y desarrollo de la matemática no fue francamente desconocido por la propia orden de la Compañía de Jesús. En esta dirección Christophorus Clavius [S.J.] (1538-1612) ${ }^{16}$, matemático y astrónomo alemán de potente influencia en toda Europa, legó tres obras decisivas no muy distante de aquella mencionada por el propio Suárez: Euclidis Elementorum libri XV (Romae, 1574), Geometria practica (Moguntiae, 1606) y Operum Mathematicorum (Moguntiae, 1611). Junto con ellas, la matemática aplicada a la física y el heliocentrismo tardaron en imponerse en forma definitiva, ${ }^{17} \mathrm{y}$ para cuando ello se consolidó, Suárez ya había publicado sus D.M. Como podrá observarse, en la época en que el Dr. Eximio publicó sus Disputationes (1597), Galileo tampoco había hecho lo propio con su Sidereus Nuncius (1610). Estos ejemplos y la obra De revolutionibus orbium coelestium (1543) de Copérnico constituyen otra arista del Renacimiento, y no su esencia efectiva. En todo caso, es justo mencionar que la Modernidad de Suárez no se consolidó en dirección a un nuevo fundamento físico y matemático de la naturaleza y la astronomía, sino de la Metafísica en un giro conceptual que alcanzará tanto la Modernidad de Descartes ${ }^{18}$ como una re-definición de las ciencias en general que tampoco pasará del todo desapercibida. Y así puede afirmarse que el caso de la filosofia de la matemática constituye, para el pensar del Dr. Eximio, un ejemplo de comprensión conceptual de injerencias metafisicas. Tema que aquí presentamos a continuación.

\footnotetext{
${ }^{15}$ Crombie, A.C., Historia de la ciencia, p. 116: "fue la aplicación con éxito de la matemática a la mecánica lo que cambió toda la concepción humana de la naturaleza y la que provocó la destrucción de todo el sistema de cosmología aristotélico".

${ }^{16}$ Lattis, J.M., Between Copernicus and Galileo. Christoph Clavius and the Collapse of Ptolemaic Cosmology, Chicago: The University of Chicago Press, 1994, pp. 1-12.

${ }^{17}$ Koyré, A., Estudios de historia, pp. 181-195: el origen del sistema heliocéntrico es propio del siglo XVI mientras que su consolidación pertenece por entero al siglo XVII. Sus principales figuras han sido Galileo, Descartes, Bruno, Tycho Brahe y Kepler.

${ }^{18}$ Prieto López, L.J., Suárez y el destino, pp. 4-11. Véase aquí la lenta gestación de posiciones próximas al racionalismo moderno donde la presencia de Suárez en las universidades españolas, francesas, alemanas y holandesas es decisiva. Nótese también su influencia en numerosos filósofos de la Modernidad, tales como Descartes, Leibniz, Wolff y Kant, entre otros. Además, $C f$. Suárez, F., Disputaciones Metafisicas, Madrid: Técnos, 2011, pp. 22-24 y pp. 34-48; Pereira, J., Suárez between Scholasticism and Modernity, Milwaukee: Marquette University Press, 2007, pp. 173-190; Baciero González, C., "Suárez y sus Disputationes Metaphysicae. Importancia y significación histórica", en: Arbor, v. CLIV, 628 (1998), pp. 451-471; Rábade Romeo, S., "Las Disputaciones Metafisicas de Francisco Suárez: innovación y proyección”, en: Arbor, v. CLIX, 625 (1998), pp. 45-60; Prieto López L.J. y otros, "Francisco Suárez, between Modernity and Tradition", en: Cauriensia, v. XII (2017), pp. 63-92.
} 


\section{Presencia imperativa de la metafisica y subordinación de la matemática}

Francisco Suárez expone los lineamientos generales de su entera doctrina científica en las D.M. I. Con ocasión de aclarar el objeto de la metafísica, articula el espectro completo de las ciencias especulativas por medio de sus objetos propios, sus relaciones de subordinación, alcance y legitimidad. Sobre esta base, lo primero que allí tiene lugar es la necesidad de demarcar una distinción con respecto a los ámbitos prácticos y artísticos. Por ello nos dice que "las ciencias especulativas únicamente se refieren al conocimiento de la verdad"19. De allí que la matemática, además de la física y la metafísica, se dirija y se detenga en el conocimiento de la verdad del ente. Pero esta verdad de las matemáticas está abocada a un ámbito más restringido que la sola verdad de todo el ente en cuanto tal. En efecto, "las ciencias matemáticas no tratan de la sustancia, sino de la sola cantidad" 20 . Esta es la precisión bajo la cual es considerado el ente. Eh ahí la verdad a la que está dirigida la ciencia matemática y la cuestión a determinar en lo extenso del trabajo.

El ámbito de la filosofia, que es especulativo, por sí se divide en metafísica, física y matemática. Luego, si evitamos la filosofia del arte y el conocimiento teorético de la filosofia práctica ${ }^{21}$, solo resta subrayar que lo que es especulativo por si tiene sede en lo real. En efecto, afirmar esta tesis impone un necesario alejamiento de la filosofia que piensa la praxis y un auténtico fundamento de cada una de las ciencias teoréticas en la realidad objetiva, pues allí: "todos ellos [la tradición aristotélica] distinguen una triple abstracción en las ciencias especulativas y reales, como son las tres citadas física, matemáticas y metafísica"22.

El texto señalado indica una distinción en el que se observan dos supuestos: (1) la imposibilidad absoluta de reducir la totalidad del saber a una única ciencia que colme por completo el intelecto ${ }^{23}$ "porque con solo pensar

19 Suárez, F., D.M. I, VI, 31: "speculativae solum ad veritatis cognitionem referantur".

${ }^{20}$ Suárez, F., D.M. I, I, 14: "et scientiis mathematicis, quae non agunt de substantia sed de sola quantitate".

${ }^{21}$ Suárez, F., D.M. I, VI, 33: "verumtamen, licet scientia practica in hoc differat ab speculativa, quod per se ordinatur ad opus, illa vero minime, ut suo loco dicetur, hoc tamen non excludit quin practica scientia proxime et immediate conferat alicuius veritatis cognitionem, immo id necessarium est, alioqui scientia non esset".

${ }^{22}$ Suárez, F., D.M. I, II, 13: "Qui omnes distinguunt triplicem abstractionem in scientiis speculativis et realibus, quales sunt tres supra numeratae, physica, mathematica et metaphysica".

${ }^{23}$ Suárez, F., D.M. I, II, 11: "addo deinde (quidquid sit de illis tribus scientiis, philosophia, mathematica et metaphysica, quo modo unaquaeque earum per se una sit, quod paulo post breviter attingemus), per sese incredibile esse eas omnes vere ac proprie unicam scientiam humanam esse". Cf. Suárez, F., D.M. I, II, 24: "non posse dari unam scientiam humanam, et propriis actibus humani ingenii acquisitam quae universaliter illud perficiat quoad omnia scibilia secundum 
que la mente se ocupa en las cosas naturales o matemáticas como tales, nos da ya a entender que rebasa los límites de la metafísica y se está valiendo de la filosofia o la matemática"24; (2) la aplicación de un criterio por el que sean únicamente tres ciencias especulativas y reales. Con este criterio se evita la inclusión de la lógica ${ }^{25}$, y permanece como única idea la relación que establece cada ciencia que considera al ente real con la materia. Y esa única relación es la operación mental de la abstracción, en tanto "por ahora bástenos a nosotros saber que no ha sido hallada una manera más apta de distinguir estas ciencias, y que, por otra parte, parece bastante idónea"26. Hay aquí dos cuestiones: (1) la convencida pertenencia y fidelidad de Suárez a la tradición aristotélica y (2) un posible conocimiento de toda una filosofia diferente que cuestione los principios propios del aristotelismo. En efecto, queda la impresión de que el Dr. Eximio ha tomado contacto con otros desarrollos especulativos de las ciencias -porque el único criterio común en el aristotelismo, según su sentir, es la abstracción-, y que lo habría marginado por la poca profundad argumentativa que ofrecía. A su vez, ello se nos manifiesta por contraste, puesto que no son propiamente los aristotélicos quienes exploran otros modos de división de las ciencias especulativas, y también por preferencia, en tanto es el mismo Suárez quien llama idónea tal distinción de ciencias. En concreto, "aquellas tres ciencias convienen, pues, en cierta abstracción, pues todas dirigen su consideración a las cosas tomadas en universal. Difieren, sin embargo, en la abstracción cuasi formal o precisiva de la materia" 27 .

Suárez hace foco en aquello que es común, que es coincidente con su intención de simplificar las diversas escuelas tradicionalmente aristotélicas, y que es útil a su propósito: la abstracción. Esto es lo idóneo. Este es el procedimiento correcto y adecuado para distinguir las ciencias y jerarquizarlas. En derredor de ello también habíamos afirmado que toda ciencia especulativa por

\footnotetext{
omnes rationes eorum, alioqui non solum illae scientiae speculativae philosophia, mathematica et metaphysica, sed etiam morales et rationales, ac denique omnes in unam coalescerent, quae esset adaequata perfectio intellectus humani, quod est incredibile".

${ }^{24}$ Suárez, F., D.M. I, II, 11: "quia, hoc ipso quod mens versari intelligitur circa res naturales, vel mathematicas ut sic, iam intelligitur transilire terminos metaphysicae, et philosophia vel mathematica uti".

${ }^{25}$ Novotný, D., "Suárez on Beings of Reason", en: Salas. V. y R. Fastiggi (eds.), A Companion to Francisco Suárez, Lieden-Boston: Brill, 2014, pp. 248-273.

${ }^{26}$ Suárez, F., D.M., I, II, 13: "Nunc nobis sufficiat hactenus non esse inventam aptiorem rationem distinguendi has scientias, et aliunde hanc videri satis convenientem".

${ }^{27}$ Suárez, F. D.M., I, II, 13: "Illae ergo tres scientiae in aliqua abstractione conveniunt, nam omnes considerant de rebus in universali; differunt tamen in abstractione quasi formali et praecisiva a materia”.
} 
sí se detiene en el conocimiento de la verdad del ente, que es interpretado como objeto y ahora precisado como universal. Todo objeto científico especulativo y real es considerado abstractivamente, o lo que es lo mismo, en su universalidad. Los conceptos de tales ciencias son objetivos porque referencian los ámbitos propios de consideración e indagación del ente per se, cuya máxima extensión es acorde con su universalidad, en tanto que tales entes caen bajo el objeto propio de cada ciencia. Mas, este común procedimiento de la razón se resuelve en cada una de ellas diferenciadamente, según su consideración -que depende de la razón abstractiva- de la materia.

"Se sigue lógicamente que según sea el diverso modo de abstracción, cambiará el objeto cognoscible en cuanto tal, y por ello suele decirse que esta abstracción, en cuanto tiene su fundamento en el objeto mismo, es la razón formal sub qua de tal objeto en cuanto cognoscible... Las cosas tienen tanta mayor perfección en su inteligibilidad cuanta mayor es su abstracción de la materia, y de modo parecido, el conocimiento es tanto más abstracto, cuanto es más inmaterial”28.

"Fácilmente se entiende y aprueba... que ninguna ciencia sobrepasa los limites de su objeto formal o de la razón formal sub qua de su objeto, sino que considera todo cuanto en ella se contiene" 29 .

Según el parecer del Dr. Eximio, la verdad de las ciencias que enuncia la razón queda expuesta ante la mente por abstracción, atendiendo a su razón formal sub qua en cuanto cognoscible. De ahí se sigue: (1) la operación mental de la abstracción antecede y posibilita la especificación del ente sub qua; (2) la noción de ente sub qua expresa la razón formal del ente por el cual es conocido; (3) la diferencia entre las ciencias está asentada en la abstracción. A su vez, la abstracción mira al ente en cuanto objeto de conocimiento, y, por ello, el pasaje epistémico de la consideración del ente a aquella otra del ente como objeto tiene aquí su origen. Esto es, la operación abstractiva de la razón y la posibilidad de determinar por medio de ella la diferencia entre las ciencias especulativas y

\footnotetext{
${ }^{28}$ Suárez, F., D.M. I, II, 13: "recte ex diverso modo abstractionis intelligitur variari obiectum scibile ut sic; et ideo solet dici haec abstractio, quatenus in ipso obiecto fundamentum habet, ratio formalis sub qua talis obiecti in ratione scibilis. Item, quia res eo sunt perfectius intelligibiles, quo magis abstrahunt a materia; et similiter cognitio, quo est de obiecto immaterialiori et consequenter abstractiori".

${ }_{29}$ Suárez, F., D.M. I, II, 13: "Ex hac autem recepta doctrina facile intelligitur et probatur assertio posita, quia scientia non transgreditur limites sui obiecti formalis, seu rationis formalis sub qua sui subiecti; considerat autem quidquid sub illa continetur".
} 
reales ha dado lugar a la consideración del ente como objeto o concepto objetivo, porque su anclaje con lo real continúa siendo el ente o la sustancia.

En el texto de Suárez se halla también el motivo de la jerarquía de las ciencias. En efecto, la conclusión sostenida por el Granadino afecta la totalidad de los saberes especulativos y reales de la siguiente manera. La metafísica es la ciencia más conocida por cuanto su objeto formal está máximamente distante de la materia -en razón de su absoluta ausencia, pues "abstraer de la materia según el ser no es otra cosa que poder existir verdadera y realmente en la naturaleza sin materia"30_ mientras que la física, por su consideración inteligible de la materia sensible, es la ciencia más oscura o menos clara. Y entre los extremos, la matemática al "toma[r] un camino intermedio [entre la física y la metafisica]"31 en tanto "abstrae conceptualmente de la materia sensible, pero no de la inteligible, ya que la cantidad, por mucho que se abstraiga, no puede concebirse más que como una cosa corpórea y material" 32 . Nuevamente, el trasfondo es la abstracción como operación intelectual. Y así la ciencia primera, inmaterial y más clara para el intelecto es la metafísica. Le continúa la matemática por la consideración de la materia inteligible, y, finalmente, la física. Con lo cual la división de las ciencias y su jerarquización es enteramente dependiente de una consideración quoad nos ${ }^{33}$. "

Y por esto, con todo derecho, se atiende a los diversos grados de inmaterialidad o de abstracción para conocer los diversos objetos cognoscibles y las diversas ciencias"34.

Ahora bien, si la matemática es una ciencia intermedia en su claridad conceptual quoad nos, y la metafísica es aún más clara y evidente en virtud de su grado de abstracción, entonces su presencia se justifica en orden a una mayor clarificación del objeto matemático. “

\footnotetext{
${ }^{30}$ Suárez, F., D.M. I, I, 16: "nihil enim aliud est abstrahere a materia secundum esse, quam quod possit in rerum natura vere ac realiter existere absque materia".

${ }_{31}$ Suárez, F., D.M. I, II, 11: "mathematicus vero medio quodam modo procedit".

32 Suárez, F., D.M. I, II, 13: "Mathematica vero abstrahit quidem secundum rationem a materia sensibili, non autem ab intelligibili, quia quantitas, quantumvis abstrahatur, non potest concipi nisi ut res corpórea

et materialis".

${ }^{33}$ La ponderación del orden de las ciencias quoad nos sobre el orden per se de las mismas se debe a la primacía del hombre en el orden del conocimiento. A este respecto, véase Rábade Romeo, S., "La metafisica de Suárez: subjetivización", p. 148: "con Suárez se inicia, aunque sea con lentitud, el proceso de subjetivización de la metafísica. Nos parece que así sucede con la potenciación de lo cogitabile en identidad de fondo con lo possibile. Es decir, la razón humana se convierte en el fulcro sobre el que ha de apoyarse y desde el que ha de elaborarse todo quehacer metafísico".

${ }^{34}$ Suárez, F., D.M. I, II, 13: "et ideo ex diverso gradu abstractionis seu immaterialitatis recte consideratur varietas obiectorum scibilium et scientiarum".
} 
Además, no siempre es necesario que la metafisica indique inmediatamente por sí misma los objetos de cada una de las ciencias, sino que basta con que dé los principios y declare los términos de que se han de valer las otras ciencias para suponer o mostrar, en cuanto fuese necesario, sus propios objetos" 35 .

La clarificación del objeto adecuado matemático, que es el concepto objetivo de cantidad, es consistente con la presencia imperativa de la metafisica, cuyo influjo parece ser, según las palabras previas del Granadino, remoto, o bien, acorde con la siguiente sentencia, inmediato, pues: "delimitar los objetos propios de las demás ciencias es oficio de esta ciencia [metafísica]"36. En ambos casos la manera en que la metafisica procede sobre los objetos de las ciencias inferiores no referencia negativamente la relación particular que guarda con la matemática. Antes bien, la presencia metafisica es benéfica para su constitución y progreso científico en virtud de la aplicación de los primeros principios metafisicos ${ }^{37}$, puesto que: "las cosas de que tratan las matemáticas incluyen los predicados comunes y trascendentales de que la metafisica se ocupa, y los mismos principios matemáticos incluyen los metafísicos y dependen de ellos"38. "

Todas las demás ciencias frecuentemente acuden a los principios de la metafisica, o bien los suponen, para poder avanzar en sus propias demostraciones o razonamientos, con lo cual se explica que muchas veces se yerra en las otras ciencias por desconocimiento de la metafísica"39.

Aquí se expresan dos caminos. Por un lado, está la relación descendente desde la metafísica hacia la matemática. Por el otro, el camino ascendente desde la matemática hacia la metafísica. El punto de sutura que lo habilita está dado

\footnotetext{
${ }^{35}$ Suárez, F., D.M. I, IV, 12: "Ac deinde non semper necesse est ut metaphysica per seipsam immediate ostendat singularium scientiarum obiecta, sed satis est quod tribuat principia, et declaret terminos quibus aliae scientiae uti possunt ad supponenda vel ostendenda, quantum necesse fuerit, obiecta sua".

${ }^{36}$ Suárez, F., D.M. I, II, 26: "Ut autem hoc declarem, dico imprimis, metaphysicam non attingere rationem substantiae materialis, et alias similes, quae sine materia non reperiuntur, nisi quatenus illarum cognitio propria necessaria est ad tradendas generales divisiones entis in decem 302 summa genera, et alias similes, usque ad propria obiecta aliarum scientiarum praescribenda; hoc enim munus proprium est huius scientiae".

${ }^{37}$ Suárez, F., D.M. I, IV, 15: "quia prima principia ut sic sunt immediata, unde a priori demonstrari non possunt".

${ }^{38}$ Suárez, F., D.M. I, V, 23: "sic enim res illae de quibus mathematicae tractant, includunt communia et transcendentia praedicata, de quibus metaphysica disserit; principia etiam mathematica includunt metaphysica, et ab illis pendent".

${ }^{39}$ Suárez, F., D.M. I, IV, 5: "omnes enim aliae scientiae saepe utuntur principiis metaphysicae, aut ea supponunt, ut in suis demonstrationibus vel ratiocinationibus progredi possint, unde saepe contingit ex ignoratione metaphysicae in aliis scientiis errari".
} 
por las afirmaciones de Suárez en lo relativo a la fundamentación metafísica de la matemática y que es, a la vez, la razón por la cual la mentada ciencia avanza especulativamente sin error. Pero la matemática no se identifica total o parcialmente con la metafisica, pues su ayuda no impide la independencia de principios primeros y propios de cada ciencia inferior -"las demás ciencias distintas de la metafísica tienen evidencia omnimoda, apoyándose en sus propios principios" 40 en tanto "las ciencias específicas [física y matemática] consideran los objetos cognoscibles particulares bajo sus propias razones" ${ }^{\text {"1 . " }}$

No es necesario que la ciencia que estudia una razón universal descienda en particular a todas las cosas que se contienen bajo la misma, sino solo a aquellas que participan de la misma razón de cognoscible o del mismo grado de abstracción"42.

La metafísica guía las ciencias según la universalidad ${ }^{43}$ que le es propia a cada una de ellas y según su tercer grado de abstracción sin violentarse consigo misma ni violentar los objetos de las demás ciencias. Esto es, la metafisica no puede descender v.g. hasta la ciencia matemática e investigar al modo en que lo hace un matemático. Solo puede legislar con sus principios el sendero seguido por las ciencias segundas. Y lo hace en virtud de su universalidad, puesto que "mediante esta ciencia [metafísica] no conoce aquellas cosas [v.g. matemáticas] absolutamente y según sus propias razones, sino en cuanto contenidas en un universal" ${ }^{44}$. Por este motivo la ayuda brindada por la metafisica a la ciencia de las matemáticas no menoscaba el razonamiento matemático. Antes bien, lo propulsa en la dirección que la metafísica señala, impidiendo deslices o desvios que pudieran afectar la organicidad del saber en su totalidad. El progreso matemático tiene en su base los principios y los términos fijados por la ciencia metafísica sin que esto último signifique una intromisión indebida, puesto que

\footnotetext{
40 Suárez, F., D.M. I, II, 8: "Aliae scientiae a metaphysica ex propriis principiis habent omnimodam evidentiam".

41 Suárez, F., D.M. I, II, 23: "Ad scientias autem specificas spectare particularia obiecta scibilia secundum proprias rationes eorum".

${ }^{42}$ Suárez, F., D.M. I, II, 22: "Non est enim necesse ut scientia, quae considerat universalem rationem, in particulari descendat ad omnia quae sub tali ratione continentur, sed solum ad ea quae eamdem rationem scibilis, seu eamdem abstractionem participant".

${ }^{43}$ Heider, D., "Suárez on the metaphysics and epistemology of universals", en: Salas, V. y R. Fastiggi (eds.), A Companion to Francisco Suárez, Lieden-Boston: Brill, 2014, p. 190: "Suárez's metaphysics of universals is fundamentally formed by the moderately realistic conceptions of John Duns Scotus and Thomas Aquinas. In contrast to Pedro Fonseca, they all admit that universal unity is nothing more than the unity of reason with a foundation in a thing".

44 Suárez, F., D.M. I, II, 7: "Dicit autem, quodammodo, quia non scit illa simpliciter et secundum propria ex vi talis scientiae, sed quatenus sub universali continentur".
} 
"la metafísica no considera todos los entes según todos sus grados o razones especiales, tal como los considera el filósofo o el matemático"45. Cada una es independiente. Cada una tiene sus principios propios y su metodología.

Nuevamente, el conocimiento efectivo que tiene el metafisico del matemático es el que le corresponde a su grado propio de abstracción. Y con ello queda asentado que la comprensión de Suárez sobre las matemáticas es conforme con el pensamiento metafísico, pues "la metafísica no trasciende la abstracción propia de su objeto; no considera a cada ente en particular"46.

\section{Ciencias matemáticas: una dirección insospechada}

Al comienzo del apartado precedente se afirmaba que la matemática no se ocupa de la sustancia sino de la sola cantidad. También se esclareció que la abstracción es la operación racional por la cual se posibilita la diferenciación de las ciencias, siendo la matemática aquella que abstrae de la materia sensible y se mantiene en la consideración de la materia inteligible. Luego, la cantidad de la sustancia se presenta ante la razón humana como la materia inteligible de la cual se ocupa la mentada ciencia, pues "la matemática abstrae de aquella materia según la razón y no según el ser, y por eso se dice que no abstrae de la materia inteligible" ${ }^{\prime 7}$. En conformidad con ello, el correlato para el intelecto está en lo siguiente: "las ciencias matemáticas perfeccionan al entendimiento en cuanto que, de algún modo, abstrae de la experiencia de los sentidos externos, pero con dependencia todavía de la imaginación o fantasía"48.

En el marco de las tres ciencias especulativas y reales y por oposición a la matemática están la física y la metafísica. Ambas se ocupan de la sustancia. La primera, de la sustancia material y sensible, y se corresponde con el primer grado de abstracción de la materia. La segunda, del ente en cuanto ente o ente ut sic que se corresponde con el tercer grado de abstracción de la materia, ya no según la razón, sino según el ser. De acuerdo con ello, la cantidad de la sustancia, que no es ni se reduce a la propia sustancia, pertenece en términos

\footnotetext{
${ }^{45}$ Suárez, F., D.M. I, II, 8: "metaphysicam non considerare entia omnia secundum omnes gradus seu rationes speciales, prout a philosopho vel mathematico considerantur".

${ }^{46}$ Suárez, F., D.M. I, II, 28: "ideo metaphysicam non considerare in particulari omnia entia, quia non transcendit propriam abstractionem sui obiecti".

${ }^{47}$ Suárez, F., D.M. I, I, 14: "mathematica abstrahit ab illa materia secundum rationem, non autem secundum esse, et ideo dicitur non abstrahere a materia intelligibili".

${ }^{48}$ Suárez, F., D.M. I, IV, 4: "Mathematicae vero scientiae illum videntur perficere, prout aliquo modo abstrahit ab externorum sensuum experimento cum dependentia tamen ab imaginatione, seu phantasia".
} 
categoriales, a los accidentes. Por ello la abstracción de segundo grado no se efectúa sobre la sustancia en orden a la misma sustancia, sino en orden al primer accidente de la sustancia material, que es la cantidad. De alli (1) su vinculación con la ciencia física, porque la cantidad en lo real es parte constitutiva de la conformación de la sustancia, y también (2) su lugar en el orden de las ciencias, que considera la cantidad abstracta y con absolutez, base de la diferencia entre ambas ciencias.

"El accidente puede ser objeto propio de alguna ciencia. Por consiguiente, esta ciencia, que es la más universal de todas [metafísica], no considera al accidente solo como una propiedad demostrable de la sustancia, sino como algo que participa en sí mismo de la razón y propiedades del ente, aunque participe de ellas siempre en orden a la sustancia"49.

Suárez también subraya la relación entre la metafisica y la matemática en la participación del accidente respecto de la sustancia, en tanto el accidente es alcanzado por la razón y las propiedades del ente. Eh alli el núcleo que pone de manifiesto el supuesto de la ciencia de la cantidad que se enuncia y se trata en la ciencia metafísica. Es esta última la única ciencia que entiende y posibilita, por sus principios y propiedades, la existencia científica de la matemática, puesto que ella se vale de aquellos principios y propiedades no como algo de su propia ciencia y sobre los cuales versa, sino como algo supuesto sobre la que se sostiene, porque "los objetos de las ciencias inferiores no están subordinados accidentalmente al ente o la sustancia, sino por sí mismos y esencialmente, como se ve claramente en el ente natural, que es el objeto de la filosofia, y en la cantidad, que es el objeto de las matemáticas"

Y finalmente, aunque el mismo accidente sea algo propio de la sustancia, sin embargo, considerado en sí mismo, puede estudiarse a veces en él algo como en absoluto, y por eso puede alguna ciencia versar únicamente sobre accidentes, como las matemáticas, sobre la cantidad"

La consideración del accidente de la cantidad como absoluto está posibilitada tanto por la configuración de la sustancia sensible como por la operación

\footnotetext{
49 Suárez, F., D.M. I, I, 25: "Haec igitur scientia, quae un\}iversalissima est, non considerat accidens solum ut proprietatem de substantia demonstrabilem, sed ut ipsum in se participat rationem et proprietates entis, quamvis illas semper participet in ordine ad substantiam".

50 Suárez, F., D.M. I, V, 51: "Rursus obiecta inferiorum scientiarum non sunt per accidens subordinata enti aut substantiae; sed per se et essentialiter, ut patet in ente naturali, quod est obiectum philosophiae, et de quantitate, quae est obiectum mathematicae".

51 Suárez, F., D.M. I, I, 24: "Ac denique quamvis ipsum accidens aliquid substantiae sit, tamen in illo ut sic potest interdum aliquid veluti absolute considerari; et ideo aliquando potest aliqua scientia in solis accidentibus versari, ut mathematica in quantitate".
} 
racional de la abstracción cada vez más inmaterial. Los grados abstractivos, que reflejan mayor inteligibilidad y certeza para la razón, han manifestado, según el sentir de Suárez, que la matemática ocupa el segundo e intermedio en razón de su claridad conceptual, no estrictamente dependiente de los sentidos. Conforme con ello el objeto matemático, más inmaterial y cierto que el objeto de la fisica, debería ser más noble. Pero la nobleza del objeto y la certeza del mismo no se corresponden proporcionalmente, puesto que "la filosofia natural es más noble que las matemáticas, aunque sea menos cierta, debido a la nobleza de su objeto"52.

A su vez, la subordinación de las ciencias inferiores a la metafisica obedece a la certeza que presentan ante la mente. La filosofia primera es la más cierta según su objeto adecuado, que es el ente ut sic, pues "la metafísica no hay duda de que por sí misma es certísima y que aventaja en esto a las matemáticas. Pues la certeza de una ciencia hay que ponderarla por su objeto"53. Nótese que certeza y nobleza de una ciencia dependen del objeto. Pero de ambas es la certeza la que aquí debe subrayarse, no tanto por el orden quoad nos de las ciencias, sino en cuanto que es la clave que permite progresar en el conocimiento científico. Suárez parece insistir en una comprensión de las ciencias que destaca una certeza cual resultado de la razón abstractiva.

$\mathrm{El}$ objeto de la metafísica no da respuesta satisfactoria para cuestiones no metafísicas. Su lugar entre las ciencias, que es el primero en razón de su nombre de filosofia primera, no supone una comprensión adecuada en el ámbito de las ciencias que tiene subordinadas. Estas últimas tienen su objeto propio y solo remotamente responden a la metafísica, y ello más por un análisis de la filosofia primera sobre sí misma y su relación con las ciencias inferiores. Por tal motivo la certeza de la matemática, si bien no desplaza ni reemplaza aquella que es propia de la metafísica, si relativiza su locus medieval -lo cual parece insinuar una tímida ruptura con el aristotelismo escolástico- en favor de una aproximación casi imperceptible para con los tiempos de la matemática renacentista, ya que "quizás en alguna cosa puede la metafisica humana ser más

\footnotetext{
${ }^{52}$ Suárez, F., D.M. I, V, 32: "et naturalis philosophia est nobilior quam mathematica, licet sit minus certa, propter obiecti nobilitatem".

${ }^{53}$ Suárez, F., D.M. I, V, 24: "Scientia igitur harum rerum ex sese non dubium est quin sit certissima, et quod mathematicas scientias antecellat. Nam certitudo scientiae hoc modo pensanda est ex obiecto".
} 
perfecta y cierta de lo que lo son las matemáticas" ${ }^{54}$. A favor de esto último, y de acuerdo con los nuevos aires del propio Renacimiento, el Dr. Eximio sugiere: “

Aunque la metafísica sea siempre en nosotros inferior en esta parte a las matemáticas en cuanto a su certeza, sin embargo, en una consideración absoluta y esencial es más noble, para lo cual importa mucho que de por sí y por parte de su objeto sea más cierta. Porque la dignidad de su objeto tiene una importancia grande para la dignidad de la ciencia, hasta el punto de redundar en la ciencia misma"55.

De acuerdo con las últimas consideraciones suarecianas acerca del objeto de la matemática y su grado de certeza -correlativo de su grado de abstracción-, afirma en clave barroca y renacentista lo siguiente: "las ciencias, hablando absolutamente, no suponen que su objeto existe actualmente, ya que esto... es accidental para la razón de ciencia, exceptuando la que trata de Dios"56. Por lo tanto, puede afirmarse que la matemática se ocupa del concepto objetivo de la cantidad absoluta que es la materia inteligible apta para existir en el ente material. No hay sustancia fisica que carezca de materia y de cantidad. El pensamiento suareciano antecede esa obviedad y coloca la posibilidad de la existencia física -y deductivamente, de la matemática- antes de la misma existencia ${ }^{57}$. Para el pensamiento científico que atañe a la física y la matemática es la posibilidad de la existencia real lo que antecede a la existencia real. En lo que toca a la matemática, basta con subrayar que su concepto objetivo es independiente y que efectivamente prescinde de la existencia real. El objeto de las matemáticas guarda su relación con lo real sin necesidad de su existencia, pues, efectivamente, no hay existencia fáctica de la cantidad en su absolutez. Empero ello no niega ni obstaculiza el hecho de la existencia real de las sustancias físicas y la ciencia que da cuenta de ello. El sistema científico suareciano es pensado, en atención a la totalidad de lo real y existente, en clave contingente -para las ciencias físicas y matemáticas- en relación de

\footnotetext{
54 Suárez, F., D.M. I, V, 26: "fortasse in aliquo statu posse metaphysicam humanam esse perfectiorem et certiorem quam sint mathematicae".

55 Suárez, F., D.M. I, V, 26: "quamvis metaphysica in nobis semper sit quoad hanc partem inferior mathematica in certitudine, nihilominus simpliciter et essentialiter esse nobiliorem; ad quod multum refert quod sit secundum se, et ex parte obiecti certior; nam dignitas obiecti maxime spectat ad dignitatem scientiae, et illa est quae per se redundat in scientiam".

${ }^{56}$ Suárez, F., D.M. I, IV, 10: "Scientiae autem, per se loquendo, non supponunt suum obiectum actu existere... accidentarium est ad rationem scientiae; illam excipio, quae est de Deo".

57 Suárez, F., D.M. II, IV, 7: "et sic dicimus essentiam esse realem, quae a deo realiter produci potest, et constitui in esse entis actualis... unde solum dicere possumus essentiam realem eam esse quae ex se apta est esse, seu realiter existere".
} 
dependencia con el ente necesario y de existencia siempre actual, que es Dios y cuya ciencia es la metafisica. Por ello, desde lo existente que no sea Dios (la creación), todo es contingente y, por correlación, toda ciencia que vera sobre lo real y contingente recibe la nominación de accidental. Esto es, la física y la matemática son accidentales solo por el objeto contingente del cual versan. Y se llaman contingentes tales conceptos objetivos solo por comparación con la metafisica y por la dependencia de las sustancias materiales de Dios, puesto que "aunque la ciencia no considere la existencia de la cosa en acto ejercido, considera, sin embargo, aquella en acto signado, esto es, qué sea la existencia misma y de qué modo le conviene o puede convenir a cada cosa"58.

\section{Objeto adecuado y principios matemáticos}

Las ciencias se articulan según su objeto adecuado ${ }^{59}$ en cuanto hace las veces de centro y fundamento de cada ciencia, pues "objeto adecuado es aquel subiectum del que se demuestran sus propiedades"60, o más precisamente "el subiectum de una ciencia es aquello cuyas propiedades más comunes se demuestran por sí e inmediatamente en esta ciencia"61. De allí que el subiectum de cualquier ciencia se enuncie ante la mente como objeto adecuado. En el caso de la matemática es la cantidad absoluta, donde la noción de cantidad hace las veces de fuente y principio de las propiedades más comunes que la filosofia de la matemática por si e inmediatamente demuestra. Así, del concepto de objeto adecuado de la matemática emergen demostrativamente todas sus propiedades en orden a contemplar la naturaleza cuantitativa y abstracta del accidente cantidad.

\footnotetext{
${ }^{58}$ Suárez, F., D.M. I, V, 40: "Deinde dicitur, esto scientia non consideret existentiam rei in actu exercito, considerare tamen illam in actu signato, id est, quid sit ipsa existentia, et quomodo unicuique rei conveniat, aut convenire possit".

${ }^{59}$ Courtine, J.-F., Suarez et le système de la métaphysique, París: Épiméthée, 2015, pp. 12-41. Este capitulo, dedicado al tratamiento escolástico de la metafísica como ciencia, se detiene en la transformación de la noción de subiectum en obiectum. Sobre la base de numerosos textos que recorren principalmente las posiciones de Avicena, Tomás de Aquino y Duns Scoto, se busca mostrar el giro que comienza en la teología como sacra doctrina hasta su configuración como metafisica en un horizonte cada vez más racionalista. Mientras que la noción de subiectum designa la unidad de una ciencia per se, la noción de obiectum, en cambio, siempre enmarcada en un contexto noético, expresa la relación de la ciencia según la expresión quoad nos. Allí, en su configuración, interviene activamente la inteligencia. A su vez la transformación señalada, añadimos, opera en la totalidad de las ciencias especulativas y reales, lógicas y prácticas.

${ }^{60}$ Suárez, F., D. M. I, I, 21: "obiectum adaequatum est illud subiectum de quo passiones demonstrantur".

61 Suárez, F., D. M. I, I, 28: "illud est subiectum scientiae de quo proprietates communiores in scientia immediate et per se demonstrantur".
} 
A su vez Suárez había afirmado la injerencia de la metafisica en la matemática según la sentencia que reza: "[la metafisica] distingue varios grados de entes bajo los cuales están contenidos todos los objetos de las ciencias."62 Ahora bien, las propiedades comunísimas demostradas por sí e inmediatamente en la matemática no deben contradecir los principios primeros de la metafísica, ${ }^{63}$ y aún más, estos últimos deben trascender el saber de las cantidades matemáticas, haciéndose también presentes en ellos.

"Porque hay efectivamente dos grupos de principios en las ciencias... Unos propios, que explica cada ciencia en particular, y otros comunes a varias ciencias, o mejor, a todas ellas, ya que todas se valen de ellos en cuanto lo exige su objeto, y en cuanto que de ellos dependen todos los demás principios particulares suyos... por consiguiente, como todas las ciencias dependen en sumo grado de estos principios, han de ser perfeccionadas necesariamente por la metafisica" 64 .

"La matemática tiene principios propios evidentes, conocidos por sí mismos, basándose en los cuales desarrolla sus demostraciones evidentes, sin apoyarse en testimonio alguno o en fe humana, como es claro, sino únicamente en la evidencia que fuerza al entendimiento. Tampoco se apoya en los sentidos, pues absolutamente hablando, abstrae de la materia sensible, y por consiguiente, de los efectos sensibles. Y si alguna vez usa de figuras visibles, lo hace solo para que la mente capte los términos de los principios y pueda penetrar en la conexión intrínseca de estos"65.

Demos un paso más. Los principios de la metafisica direccionan los principios de la matemática. En ocasiones los corrigen cuando se trata de principios que contradicen el saber metafísico. Pero en ningún caso se superponen o reemplazan o confunden. Son ciencias diversas con objetos adecuados dife-

\footnotetext{
${ }^{62}$ Suárez, F., D. M. I, IV, 10: "et deinde distinguendo varios gradus entium, sub quibus omnia obiecta scientiarum continentur".

${ }^{63}$ Suárez, F., D. M. I, IV, 5: "secundo vero addendum est scientiam metaphysicae non solum propter seipsam esse convenientem, sed etiam ad alias scientias perfecte acquirendas esse valde utilem... metaphysicam solam versari circa prima principia reliquarum scientiarum".

${ }^{64}$ Suárez, F., D. M. I, IV, 5: "Sunt enim duplicia principia scientiarum, ut in eodem libro docet, c. 8: Quaedam propria, quae in unaquaque scientia declarantur; alia communia multis, vel potius omnibus scientiis, quia omnes illis utuntur, ut res subiecta postulat, et quatenus ab eis caetera particularia principia pendent".

${ }^{65}$ Suárez, F., D.M. I, II, 8: "nam mathematica habet propria principia evidentia et per se nota, ex quibus per evidentes demonstrationes procedit, nec nititur aliquo testimonio aut fide humana, ut per se notum est, sed evidentia cogente intellectum. Neque etiam nititur sensu, nam, per se loquendo, abstrahit a materia sensibili, et consequenter ab effectibus sensibilibus. Quod si aliquando utitur figuris visibilibus, solum est ut mens perfecte apprehendat términos principiorum, ut possit intrinsecam eorum connexionem perspicere".
} 
rentes y principios distintos. Ambos conjuntos de principios son universales en la región de entes que considera. Y esos principios, cualquiera sea la ciencia, son dobles: "unos se llaman complejos o compuestos, como son los que sirven para establecer la demostración; otros son simples, y están representados por los términos que hacen el papel de medio de la demostración a priori. Los primeros se llaman principios del conocimiento. Los otros, principios del ser"66.

Ahora bien, los principios rectores y primeros de la ciencia matemática son para ella evidentes en virtud de su fundamentación racional. Es la razón la que actúa en la organización del saber científico, fijando su objeto adecuado y mostrando cómo emergen desde allí los principios primeros y propiedades. Una vez más las ciencias y su jerarquía se manifiestan quoad nos. Pero el avance de la razón en la constitución de la matemática -o de cualquier otra ciencia- no puede quedar fundada en la autoridad humana. De hecho, si la ciencia se funda exclusivamente en la autoridad humana, no hay verdaderamente ciencia: hay una reliquia de un saber científico de alguna época pasada. Un detalle más. Suárez claramente se reconoce deudor de la ciencia del pasado, y especialmente de los argumentos filosóficos de cuño aristotélico medieval y escolástico. Pero esas autoridades -v.g. Proclo, Tomás de Aquino, Cayetano- nada valen si la razón no las ha vuelto a pensar. Solo el pensamiento que las piensa vuelve a revitalizar los argumentos del pasado con una libertad diferenciante. Y esta es la clave por la que la autoridad humana vale en la ciencia. Pero el esfuerzo de volver a pensar tales argumentos solo se ajusta al nivel que le corresponde: una autoridad humana en ciencia es una sugerencia que señala un sendero. Y por ello se la llama opinión para cada saber especulativo racional, mientras que recibe el nombre de opinión de fe para el caso de la teología. De allí se sigue que la única manera de revitalizar el pensamiento de épocas pasadas provenga de la distinción de principios del conocimiento que actúan en las demostraciones, revisando y pensando los argumentos ya existentes en ciencia. Pero ello parece imposible si antes no están claros los términos para una demostración a priori donde queden reflejados los principios del ser. Por ello, subraya Suárez, los principios trascendentales del ente se hallan en los cimientos de la matemática, o lo que es lo mismo, por la radical constitución metafisica de todo ente cuantitativo, que en tanto abstracto, es estudiado por la matemática. Así queda

\footnotetext{
${ }^{66}$ Suárez, F., D.M. I, I, 29: "quaedam dicuntur complexa seu composita, qualia sunt illa ex quibus demonstratio conficitur; alia sunt simplicia, quae significantur per terminos qui loco medii in demonstratione a priori sumuntur. Priora dicuntur principia cognitionis; posteriora autem, principia essendi".
} 
claro que los principios metafísicos orientan el matemática según el propio avance racional sobre el ente cuantitativo, abstraído y absoluto, revisando sus demostraciones -el modo de demostrar, mientras que el contenido es específicamente matemático- y sus términos -donde aparecen los trascendentales y la raíz esencial de la no contradicción y la aptitud para la existencia-67.

\section{Conclusión}

Francisco Suárez, auténtico precursor de la Modernidad, ha operado una transformación profunda del pensamiento escolástico medieval y neoescolástico renacentista. Su entera doctrina de la ciencia es barroca y su giro especulativo en ciencias señala el traspaso y paulatina sustitución de la noción de genus subiectum a aquella otra de obiectum adaequatum. Aquí se precisa una novedad radical que debe siempre subrayarse y que en el caso de la matemática se presenta enteramente conceptual y esencialmente objetiva en virtud de la ponderación de la razón abstractiva, la orientación metafisica y el orden quad nos de las ciencias.

La ciencia de la matemática en su conjunto, según la concepción impresa por el Estagirita y acorde con tal tradición, se orienta a la contemplación de la verdad del ente en cuanto cantidad absoluta. Suárez encauza la misma ciencia por una vía que no parece ser del todo medieval y que paulatinamente se integraría con el pensamiento de la matemática moderna. Hay en el Granadino un movimiento de traslación del lugar que aristotélicamente ocupaba la ciencia matemática hacia uno que tímidamente lo cuestiona. De la recepción del saber medieval hacia la modernidad Suárez representa un eslabón sugerente que se inclina sutil -y no abiertamente- hacia los aires de una matemática nueva tanto por su lugar en el orden de las ciencias cuanto por su progreso y capacidad de comprensión de la naturaleza.

El fundamento abstracto y conceptual objetivo de la matemática, que es la cantidad absoluta, continuó sin embargo en la posición de ciencia segunda acorde con su grado de abstracción. La extensión de su certeza hacia otros ámbitos científicos constituye la efectiva clave que propiciará, con todo, la previa

\footnotetext{
${ }^{67}$ Esposito, C., Suárez, filósofo barroco, p. 39: "estos momentos de la doctrina metafísica suareciana -la reductio craturae ad entem e l'aptitudo entis ad existendum- deben considerarse siempre unidos. Reducir la creatura a ente es la estrategia para encontrar en el ente mismo la tendencia constitutiva a ser, esto es, a existir. La aptitudo es como la huella dinámica de la creaturidad pensada como orden trascendental".
} 
afirmación sobre el pasaje epistémico desde el medioevo hacia la modernidad matematizante.

El orden de las ciencias especulativas y reales parece firmemente reflejado quoad nos. La ponderación de la razón abstractiva y la certeza consiguiente lo confirmarían. Por medio de la abstracción que se lleva a cabo en la filosofía de las matemáticas se procede aprehendiendo abstractivamente el accidente de la cantidad respecto de la sustancia singular para dar lugar al concepto objetivo que expresa su objeto adecuado como concepto de cantidad absoluta.

El orden científico quoad nos, manifestado por el orden ascendente de las tres ciencias especulativas gracias a la abstracción, también ha puesto en evidencia la relativa claridad que representa para la razón la ciencia de la matemática. Y aquí hemos destacado algunos detalles: (1) auxilio de la metafisica con vistas a colaborar en la conformación del objeto adecuado de la matemática; (2) precisión de su ayuda, centrada en tres ítems: (a) orientación última metafísica de todo conocimiento; (b) clarificación de los términos matemáticos en relación con aquellos de la metafísica; y (c) aplicación de los primeros principios metafísicos.

Recibido: $19 / 08 / 2020$

Aceptado: 26/05/2021

\section{Bibliografia}

Aquinatis, T., Expositio libri posteriorum, 2da. edición, Roma-París: Commissio Leonina-j. Vrin, 1989.

Aquinatis, T., In duodecim libros metaphysicorum aristotelis expositio, Marietti, TauriniRomae, 1971.

Aquinatis, T., In libros aristotelis de caelo et mundo expositio, Ex typographia polyglotta s. c. de propaganda fide, Romae, 1886.

Baciero González, C., "Suárez y sus Disputationes Metaphysicae. Importancia y significación histórica”, en: Arbor, v. CLIV, 628 (1998), pp. 451-471. https://doi. org/10.3989/arbor.1998.1628.1763

Belda Plans, J., La escuela de Salamanca y la renovación de la teología en el siglo XVI, Madrid: BAC, 2000.

Courtine, J.-F., Suarez et le système de la métaphysique, París: Épiméthée, 2015.

Crombie, A.C., Historia de la ciencia: de san Agustin a Galileo/2: siglos XIII-XVII, Madrid: Alianza Editorial, 1987.

Esposito, C., "Suárez, filósofo barroco", en: Cauriensia, v. XII (2017), pp. 25-42.

Fabro, C., Historia de la filosofia I, Madrid: Rialp, 1965. 
Florido, F.L., "Una estructura filosófica en la historia de la filosofia", en: Anales del seminario de historia de la filosofia, 17 (2000), pp. 195-216.

Florido, F. L., Las filosofias en la Edad Media. Crisis, controversias y condenas, Madrid: Biblioteca Nueva, 2010.

Gilson, E., El ser y los filósofos, Pamplona: Eunsa, 1979.

Gilson, E. y Th. Langan, Filosofía moderna, Barcelona: Emecé, 1963.

Hale, J.R., La Europa del renacimiento 1480-1520, Madrid: Siglo XXI, 2016.

Heider, D., "Suárez on the metaphysics and epistemology of universals", en: Salas, V. y R. Fastiggi (eds.), A Companion to Francisco Suárez, Lieden-Boston: Brill, 2014, pp. 164-191. https://doi.org/10.1163/9789004283930_008

Koyré, A., Estudios galileanos, España: siglo XXI, 2009.

Lattis, J.M., Between Copernicus and Galileo. Christoph Clavius and the Collapse of Ptolemaic Cosmology, Chicago: The University of Chicago Press, 1994. https:// doi.org/10.7208/chicago/9780226469263.001.0001

Lohr, Ch.H., "Del aristotelismo medieval al aristotelismo renacentista", en: Patristica et Mediaevalia, v. XVII (1996), pp. 3-15.

Novotný, D., "Suárez on beings of reason", en: Salas. V. y R. Fastiggi (eds.), A Companion to Francisco Suárez, Lieden-Boston: Brill, 2014, pp. 248-273. https://doi. org/10.1163/9789004283930_011

Parker, G., Europa en crisis 1598-1648, Madrid: Siglo XXI, 2017.

Pereira, J., Suárez between Scholasticism and Modernity, Milwaukee: Marquette University Press, 2007.

Poncela González, Á., "Aristóteles y los jesuitas. La génesis corporativa de los cursus philosphicus”, en: Cauriensia, v. VI (2011), pp. 65-101.

Prieto López, L.J., Suárez y el destino de la metafisica, Madrid: BAC, 2013.

Prieto López L.J. y otros, "Francisco Suarez, between Modernity and Tradition", en: Cauriensia, v. XII (2017), pp. 63-92.

Rábade Romeo, S., "Las Disputaciones Metafisicas de Francisco Suárez: innovación y proyección”, en: Arbor, v. CLIX, 625 (1998), pp. 45-60. https://doi.org/ 10.3989/ arbor.1998.i625.1788

Rábade Romeo, S., "La metafísica de Suárez: subjetivización y dinamismo”, en: Cuadernos salmantinos de filosofia, 30 (2003), pp. 145-156. https://doi.org/10.36576/ summa.1176

Salas V. y R. Fastiggi, "Francisco Suarez, The Man and His Work", en: Salas V. y R. Fastiggi (eds.), A companion to Francisco Suárez, Lieden-Boston: Brill, 2014, pp. 1-28. https://doi.org/10.1163/9789004283930_002

Suárez, F., Disputaciones Metafísicas, edición bilingüe, Rábade Romeo, S., Caballero Sánchez, S. y A. Pigcerver Zanón (trads.), Madrid: Gredos, 1960-1966.Suárez, F., Disputaciones metafísicas, Madrid: Técnos, 2011.Verdú Berganza, I., "Aspectos generales del pensamiento en el siglo XIV", en: Anales del seminario de historia de la filosofia, 10 (1993), pp. 195-208. 